\title{
PROFESORAS UNIVERSITARIAS Y CARGOS DE GESTIÓN
}

\author{
Marina Tomàs Folch* \\ Maria del Mar Duran Bellonch* \\ Cristina Guillamón Ramos* \\ José Manuel Lavié Martínez** \\ *Universidad Autónoma de Barcelona \\ **Universidad de Sevilla
}

\section{RESUMEN}

En este artículo sintetizamos los hallazgos más relevantes de una investigación exploratoria que pretende estudiar la gestión universitaria en relación a la variable género. El estudio analiza el papel de las mujeres en las estructuras horizontales y verticales de gestión de cuatro universidades catalanas, prestando especial atención a sus expectativas y percepciones. En su conjunto, los resultados del estudio identifican la existencia de dificultades tanto externas a la propia mujer, como internas a ella, que nos indican posibles explicaciones sobre la escasa presencia del género femenino en la gestión universitaria. Entre las dificultades externas, la cultura organizativa de la universidad aparece como un elemento resistente al cambio que fomenta un entorno predominantemente dominado por valores masculinos y que puede justificar la auto-exclusión de algunas mujeres de la gestión universitaria. Asimismo, el estudio también identifica los principales atributos con los que las mujeres participantes describen su propio rol en la gestión y cómo creen que éste contribuye a un liderazgo eficaz en la universidad.

Palabras clave: Gestión universitaria, género, liderazgo, cultura organizativa.

\section{ABSTRACT}

In this article we summarize the most relevant findings from a research that purports to incorporate a gender perspective to the study of university management. The study analyzes the role of women in both horizontal and vertical management structures in four Catalan universities, paying attention to their expectations and perceptions. Overall, the results point to the existence of both external and internal barriers that probably explain the lack of women in university management. Among these barriers, organizational culture appears as a change-resist- 
ant element that promotes an environment dominated by male values and could justify most women's self-exclusion from university management. The study also identifies the main features whereby these women depict their experience in management and attempt to articulate an alternative discourse for leadership in university.

Key words: University management, gender, leadership, organizational culture.

\section{Introducción: la universidad desde una perspectiva de género}

Los estudios de género se han convertido en las últimas décadas en un tópico de interés creciente dentro del ámbito de la investigación organizativa. Como sostienen Calás y Smircich (2006) en su reciente revisión sobre el tema, a pesar de que su impacto en la práctica es aún limitado, el género sigue manteniendo una importancia nuclear en tanto que categoría de análisis para el estudio de las organizaciones. En el caso de las organizaciones educativas, los estudios de género han conocido un desarrollo considerablemente menor, y sólo en la última década hemos asistido a una cierta sistematización en el análisis de las relaciones entre género, poder y cultura organizativa. Las universidades, consideradas tradicionalmente espacios para la investigación antes que un objetivo de investigación en sí mismas, han quedado en gran parte al margen de este esfuerzo de análisis.

Una simple mirada a las universidades en términos estructurales nos sugiere, sin embargo, que hay algo problemático en esta ausencia. Si revisamos las últimas estadísticas referidas a las universidades catalanas, podemos constatar que si bien las mujeres constituyen un grupo mayoritario en los claustros de educación infantil, primaria y secundaria, tan sólo representan el 34\% del profesorado universitario. Dentro de éste, además, el porcentaje va decreciendo a medida que ascendemos en categoría profesional hasta quedar en un $17 \%$ en el caso de las catedráticas de universidad. Y si nos interesamos por la representación femenina en lo que respecta a cargos académicos de gestión, las diferencias en este ámbito son aún más notorias.

En este artículo sintetizamos los hallazgos más relevantes de un estudio que pretende contribuir, en la medida de lo posible, a cubrir este vacío incorporando una perspectiva de género al estudio de la gestión en las universidades. No obstante, y de acuerdo con el enfoque adoptado, entendemos el género de forma amplia, como una dimensión constitutiva de las mismas organizaciones, que no puede obviarse en el análisis de su cultura. Sin ir más lejos, Alvesson y Billing $(1992,1997)$ evidencian el papel clave del género (masculino en este caso) en la definición de los intereses, los valores y los patrones de comportamiento vigentes en las organizaciones de la sociedad occidental actual. Así, partimos de la premisa de que el género ha actuado, y actúa, en nuestro medio como norma implícita sobre lo que es valioso y lo que no, fomentando el predominio de ciertos rasgos culturales asociados tradicionalmente a lo masculino, tales como: la eficiencia, la competitividad, el elitismo, la autonomía o la promoción de la independencia (van Vianen y Fischer, 2002).

En el artículo, en primer lugar revisamos literatura de interés para nuestro objeto de estudio, prestando una especial atención a aquellos trabajos que dentro y fuera de las or- 
ganizaciones educativas se han interesado por analizar las dificultades que experimentan las mujeres en la asunción de puestos de gestión y por describir la forma en que ellas dirigen, una vez acceden a cargos con poder. A continuación describimos los objetivos de la investigación que presentamos y ofrecemos una breve síntesis sobre el diseño metodológico adoptado. La siguiente sección presenta los resultados más relevantes obtenidos a partir de los análisis cualitativo y cuantitativo de la información y finalmente, concluimos con una discusión en la que sintetizamos los resultados más relevantes del estudio en referencia a los objetivos planteados, articulando algunas implicaciones que se derivan de los mismos y proponiendo posibles líneas de investigación futura.

\section{Cultura organizativa y género: explorando las relaciones}

El blindaje que las teorías organizativas han mostrado tradicionalmente hacia las cuestiones de género (Calás y Smircich, 2006; Halford y Leonard, 2001; Riehl y Lee, 1996) ha descansado sobre la asunción de que las culturas organizativas son neutrales en lo que al género se refiere. Desde los años 90, sin embargo, diversos estudios etnográficos han puesto de manifiesto los modos sutiles en que las culturas organizativas reproducen los valores androcéntricos. Los trabajos de Acker (2000), Gherardi (1995) o Maddock (1999) son representativos a este respecto, ilustrando cómo las culturas organizativas reflejan en sus símbolos, metáforas, artefactos, rituales, creencias y valores, la hegemonía patriarcal que opera en la sociedad más amplia.

Como señalábamos en la introducción, los estudios que se han interesado por analizar las relaciones entre cultura organizativa y género en el contexto universitario son escasos. La mayoría de evidencias disponibles, no obstante, coinciden en señalar el carácter tácito con el que las culturas universitarias reproducen pautas y creencias que pueden dificultar la plena incorporación de la mujer (Davies y Holloway, 1995).

La investigación sobre género y cultura, sin ser suficientemente significativa en su desarrollo, ha contribuido, no obstante, a iluminar las dificultades que muchas mujeres experimentan en su desarrollo profesional, en su incorporación a puestos de responsabilidad organizativa y una vez en ellos, en su progresión. En particular, los resultados de la investigación han servido para establecer el carácter cultural de muchas de éstas dificultades, consideradas "barreras" para algunos autores como Rutherford (2001). Las analizamos con más detalle en el siguiente apartado.

\section{Dificultades para el acceso de la mujer a puestos de gestión}

La investigación en torno al Ilamado 'techo de cristal' ha puesto de manifiesto las múltiples dificultades -con frecuencia invisibles o tácitas- con las que se encuentran muchas mujeres en su progresión en la escala organizativa (Powell, 1999). En el contexto universitario las cifras son concluyentes, -el género persiste como criterio diferenciador de poder. Aún así, los trabajos que han tratado de indagar en las causas que subyacen en dicho fenómeno son escasos.

Los estudios que han tratado de esclarecer los motivos de la escasa presencia femenina en los puestos de alta gestión, distinguen entre dificultades visibles y dificultades invisibles o inconscientes (Nicolson, 1997) y entre obstáculos de carácter contextual y obstáculos de carácter cultural (Hawley et al., 1998). Adoptamos en nuestro trabajo y a 
efectos heurísticos la primera de las clasificaciones comentadas. Así hablaremos de dificultades externas a la persona (psico-socio-culturales) y dificultades internas a ella (psico-socio-educativas).

Dentro del primer grupo de dificultades (las externas) se incluyen una serie de obstáculos, entre los que suelen ocupar un lugar destacado los referidos a las dificultades de conciliación entre vida personal, familiar y profesional. En el caso del desarrollo profesional en la universidad, diversos estudios apuntan el carácter relevante aunque no concluyente del comentado obstáculo (Carreño et al., 1998; Morley, 2004).

Otra suerte de dificultades externas comúnmente citadas por la literatura especializada, guarda relación con lo que Maier (1999) define como 'subestructura masculina de la organización' y, en términos generales, con la cultura androcéntrica o patriarcal que continúa definiendo implícitamente el funcionamiento de muchas organizaciones, entre ellas las universitarias. El estudio de Kettle (1996) sobre la carrera académica en universidades británicas, por ejemplo, pone en evidencia la tensión que experimentan muchas mujeres entre el modelo supuestamente igualitario en el que se mueven y una serie de valores implícitos asociados a su inferioridad que se manifiestan a través de procesos de cooptación, criterios de promoción y, sobre todo, los estereotipos de género, presentes aun hoy en día, aunque cada vez más puestos en cuestión. En este sentido, diversos trabajos señalan la influencia que ejercen algunas de estas determinadas creencias asociadas al género, desincentivando la presencia femenina en puestos de responsabilidad. Algunas de estas creencias son las asociadas a la falta de ambición femenina (Fisher, 2000; Hudson y Williamson, 2002) o a la falta de capacidad de liderazgo de las mujeres (Schein, 2001).

El segundo grupo de dificultades, las de carácter interno, se refiere fundamentalmente al papel que desempeña la interiorización de modelos conductuales diferenciados en función del sexo como producto de la socialización. Así, los valores educativos suelen premiar en la mujer el servicio antes que la ambición de poder; la sumisión antes que la capacidad de oposición. Fruto de la interiorización de tales valores, muchas mujeres experimentan lo que la literatura suele referir como el 'síndrome del camaleón', que propicia cierta negación de los propios deseos y pensamientos a favor de un comportamiento sobreadaptado y orientado a la aprobación de los otros (Maddock, op.cit).

De forma relacionada, la ausencia de modelos femeninos más o menos establecidos en los que poder reflejarse (Johnsrud, 1994; Morley, 2004) y el escaso interés que muchas mujeres sienten hacia los modelos tradicionales de liderazgo (van Vianen y Fischer, 2002) pueden estar a la base de la tendencia al rechazo femenino por los puestos de responsabilidad. Estas consideraciones, no obstante, introducen la cuestión fundamental de las relaciones entre género y liderazgo, en torno a la cual resulta difícil proponer algún argumento concluyente. En el siguiente apartado realizamos una breve revisión de esta cuestión, tratando de evitar las posiciones polarizadas con las que tradicionalmente ha sido abordado en la literatura de género.

\section{¿Existe un estilo de liderazgo femenino?}

Muchos de los estudios sobre las relaciones entre género y liderazgo han abordado el tema desde una perspectiva más bien reduccionista, de acuerdo con la cual el género se 
ha entendido más como una variable de análisis que como un marco analítico o una categoría organizadora del análisis de la realidad institucional (Calás y Smircich, 2006) e incluso algunos autores cuestionan si realmente existe un estilo femenino de liderazgo (Billing y Alverson, 2000). La principal preocupación desde esta óptica se ha definido en términos de un debate que se interroga por las posibles diferencias de sexo en procesos organizativos tradicionales como el liderazgo. En consecuencia, las posiciones adoptadas en dicho debate han tendido a quedar definidas en términos dicotómicos dentro del continuo definido por los polos igualdad/diferencia entre lo masculino y lo femenino.

El extremo que reclama la igualdad de estilos en función del sexo, auspiciado por el feminismo liberal (Alvesson y Billing, 1997), ha desembocado con frecuencia en propuestas asimilacionistas de los modelos masculinos establecidos orientados a 'arreglar a las mujeres ' (Ely y Meyerson, 2000) para que desarrollen las destrezas y estilos que se consideran necesarios para el éxito. Por su parte, el extremo que ensalza la diferencia femenina como 'valor añadido' al liderazgo ha conseguido un mayor desarrollo en la literatura especializada, si bien la base empírica que lo avala es escasa y poco concluyente (Alimo-Metcalfe, 1995; Eagly y Johnson, 1990; Riehl y Lee, 1996). De acuerdo con esta posición, las características comunales o emocionales que suelen asociarse con el género femenino, constituyen parte del bagaje diferencial que las mujeres introducen en el ejercicio del liderazgo, que quedaría así definido por un estilo de carácter más democrático, interactivo, orientado a las relaciones interpersonales y, de acuerdo con los modelos del Ilamado 'nuevo' liderazgo (Hunt, 1999), más transformacional (Burke y Collins, 2001; Eagly y Johannessen-Schmidt, 2001; Appelbaum, Audet y Miller, 2003).

La literatura educativa ha reproducido este debate con cierto mimetismo, desarroIlando argumentos que tienden a reforzar la existencia de un estilo 'femenino' distintivo en el ejercicio del liderazgo educativo Diéz Gutiérrez y otros (2005).

Las críticas en torno a la polarización a la que ha estado sometido este debate están bien articuladas en la literatura (Billing y Alvesson, 2000) y no las reproduciremos aquí. Desde nuestra óptica, en cualquier caso, aun reconociendo cierto mérito en la idea de un estilo 'femenino' en tanto que portador de nuevos modos de hacer y entender el liderazgo, somos conscientes también de los riesgos que comporta una lectura esencialista del mismo que, considerando a las mujeres como una categoría homogénea pueda contribuir a perpetuar los estereotipos de género y las estructuras de división del trabajo.

De acuerdo con la perspectiva adoptada en esta investigación, por tanto, el estudio de las relaciones entre género y liderazgo necesita una aproximación más comprensiva, que no equipare el sexo con el género, ni reduzca este último a una variable más en el entramado organizativo, sino que sirva para ofrecer análisis que incidan en la noción de género como construcción social que afecta directamente a la esencia de las organizaciones.

Coincidimos con autores como Blackmore $(1996,1999)$ que consideran que la principal aportación de la perspectiva de género al estudio del liderazgo educativo no debe ser la constatación de las diferencias, pero entendemos que si nuestro foco de interés son las mujeres y la gestión en contextos educativos, sí es necesario investigar si ellas siguen percibiendo dichas diferencias y las dificultades comentadas, ya que si ello es así, significará que el género entendido como variable diferencial, lo sea o no, sigue ejer- 
ciendo influencia en la configuración de la cultura organizativa, con todas la implicaciones que ello conlleva.

\section{Objetivos y diseño metodológico}

El objetivo general de la investigación es estudiar las experiencias y percepciones de las mujeres en el ejercicio del liderazgo en las organizaciones universitarias. Para ello se han tomado en consideración mujeres que estaban ejerciendo cargos según área de conocimiento y universidad, sin tener en cuenta otras variables como origen, clase social, raza o religión.

La investigación se planificó según dos fases diferenciadas, cada una de las cuales con objetivos específicos y planteamientos metodológicos diferentes.

La primera fase, de tipo descriptivo-extensivo, consistió en analizar la presencia y las oportunidades de promoción de las mujeres en lugares de responsabilidad en comparación con sus colegas masculinos en las cuatro universidades participantes en el estudio. Asimismo, se trataba de esclarecer si los participantes en la investigación percibían diferencias vinculadas al género en los estilos de gestión.

Concretamente, los objetivos específicos que perseguía esta fase eran los siguientes:

- Describir la distribución de cargos en las estructuras horizontales y verticales de las universidades participantes en función del género, e

- Indagar las oportunidades de desarrollo profesional de las directivas en las universidades.

Para obtener información relativa al primero de los objetivos analizamos la información publicada en la web institucional de cuatro universidades catalanas (tres públicas y una privada) durante el curso académico 2005-06, mientras que para obtener los datos referentes a los otros dos objetivos elaboramos un cuestionario ad hoc.

Una vez construido el instrumento, fue validado según la técnica de jueces y posteriormente lo aplicamos en cinco departamentos dirigidos por una profesora, seleccionados al azar entre las cuatro universidades participantes. En esta selección se tuvo en cuenta, entre otras variables, la representación de las distintas áreas de conocimiento. En total, obtuvimos respuesta de 150 profesores y profesoras (el $33.48 \%$ del profesorado adscrito a los departamentos que participaron en este estudio para el curso 2005-06).

Cuando la información estuvo codificada y la matriz de datos elaborada, el análisis estadístico lo realizamos a través del paquete informático de procesamiento de datos estadísticos SPSS versión 13.0 para Windows, mediante el cual obtuvimos la fiabilidad y validez del instrumento y los estadísticos descriptivos e inferenciales. Fruto de este análisis se puede afirmar que el cuestionario es fiable (.887 según Alpha de Cronbach) y válido (validez próxima al 75\%).

La segunda fase de la investigación, de tipo intensivo, la orientamos específicamente a explorar las experiencias de las profesoras en los cargos de gestión en la universidad.

Los objetivos específicos en este caso eran: 
- Analizar las dificultades -internas y externas- presentes en la trayectoria de desarrollo profesional de mujeres que ocupan posiciones de liderazgo educativo en diferentes contextos universitarios,

- Explorar las percepciones de las directivas sobre su comportamiento de liderazgo y los efectos que éste produce, y

- Describir las destrezas y los atributos de liderazgo que las mujeres que lo ejercen consideran más importantes para su trabajo.

Para desarrollar esta fase mantuvimos una serie de entrevistas en profundidad semiestructuradas con una muestra de profesoras que ocupaban cargos de responsabilidad en las cuatro universidades participantes seleccionadas en base a criterios de representatividad, de manera proporcional con la distribución según sexo y ámbitos de conocimiento en la universidad. En total, realizamos 18 entrevistas, de las cuales 4 a cargos de primer nivel (rectoras y vicerrectoras), 8 de segundo nivel (decanas, directoras de departamento y delegadas de rector/a) y 6 de tercer nivel (vicedecanas, jefa de estudios y coordinadoras de servicios).

Las entrevistas fueron registradas en audio y transcritas literalmente. A partir de la trascripción elaboramos una síntesis narrativa del contenido de cada una de ellas subrayando los tópicos abordados, identificando temas emergentes y capturando patrones de respuesta para cada una de las preguntas.

Posteriormente, definimos un sistema de códigos (Miles y Huberman, 1994) para facilitar la reducción y el análisis de los datos.

Para facilitar el análisis del contenido de las entrevistas utilizamos el programa informático de análisis cualitativo AQUAD Five para Windows.

Aunque la información de la primera y la segunda fase la recogiésemos de forma independiente, en tanto que respondía a unos objetivos específicos y un diseño metodológico diferentes, posteriormente los resultados obtenidos a través de distintos instrumentos y con distintos participantes los triangulamos para dar respuesta al objetivo general de la investigación.

\section{Resultados más relevantes del estudio}

Como comentábamos en el punto anterior, para indagar sobre los objetivos del estudio hemos recabado información a través de un cuestionario y de varias entrevistas.

\section{Resultados de la primera fase del estudio relativos al cuestionario}

El número de cuestionarios obtenidos corresponde a una cuarta parte por cada universidad con un ligero predominio de la U-3. Los informantes son provenientes mayoritariamente de áreas de conocimiento de las Ciencias Sociales (Derecho y Ciencias Políticas) de categoría profesional de profesor titular (32\%) seguido del colectivo de personal becario (22\%). De edades comprendidas entre 30 y 39 años con una experiencia docente universitaria de entre 10 y 19 años (44\%). El 68.7\% no han desarrollado ningún cargo directivo y el $31.3 \%$ sí. 
Describiremos los resultados agrupándolos siguiendo los objetivos mencionados en el apartado anterior.

Respecto al objetivo planteado de 'Describir la distribución de cargos en las estructuras horizontales y verticales de las universidades participantes en función del género', remitimos a la tabla ํㅜ 1 (Guillamón, 2006).

Los datos constatan que sólo una de las cuatro universidades, la U-4, está presidida por una rectora, también se extrae que los rectores conforman equipos rectorales eminentemente masculinos. Así, la rectora de la U-4 ha conformado un equipo paritario, contando con una vicerrectora y dos vicerrectores en su equipo y parece que la U-1 también tiene en cuenta el sexo de los componentes de su equipo ( $40 \%$ mujeres, $60 \%$ hombres), pero no así en el resto de universidades puesto que en el caso de la U-3 y la $\mathrm{U}$-2 el porcentaje de vicerrectores es próximo al $80 \%$.

En cuanto al objetivo de 'indagar las oportunidades de desarrollo profesional de las directivas en las universidades', se ha preguntado por los motivos de ausencia de la mujer en puestos de alta responsabilidad. Los resultados han sido los siguientes (de mayor a menor importancia): 'La atención a la familia y a la casa', 'Las normas de juego imperantes en nuestra sociedad que dejan a la mujer en inferioridad de condiciones', 'Los procesos de socialización como condicionante importante', 'Los estereotipos femeninos', 'La posibilidad de quedar embarazada' y 'La falta de confianza en sí misma'.

A la pregunta por los motivos para acceder a los cargos directivos, las respuestas están todas ellas relacionadas con el propósito de servicio a lo colectivo, mientras que los motivos relacionados con incentivos económicos, de reducción de horas de clase u otros son los menos considerados.

Sobre la disposición a asumir cargos, ninguna de las mujeres que contestan al cuestionario afirma desear cargos de primer nivel y sólo alguna de ellas aceptaría algún cargo de segundo o tercer nivel. Las razones que manifiestan por las cuáles no han ejercido ningún cargo, giran alrededor de tener otras prioridades como la investigación, la familia, etc.

\section{Resultados de la segunda fase del estudio relativos a las entrevistas}

Las entrevistas nos han permitido ahondar en aspectos más culturales y personales, complementarios al cuestionario, que permiten aportar la siguiente información respecto del objetivo 'Analizar las dificultades -internas y externas- presentes en la trayectoria de desarrollo profesional de mujeres que ocupan posiciones de liderazgo educativo en diferentes contextos universitarios'.

Las profesoras universitarias entrevistadas en este estudio han mostrado, en su mayoría, no solo una conciencia de ser vistas como 'otras' en el contexto académico de la universidad, sino también de haber estado sujetas a procesos de evaluación y promoción construidos en referencia a una norma 'masculina' que las discriminaba abiertamente.

Entre las dificultades externas para el desarrollo profesional sin duda, la que más han reconocido las mujeres en el desarrollo de su carrera académica en la universidad, se relaciona con el conflicto de roles que experimentan al tener que atender simultánea- 
mente las demandas familiares y las profesionales. De hecho, éste constituye, de acuerdo con las participantes 'el handicap más importante' (ent. 004, 351) y 'el gran problema de la mujer' (ent. 005, 311).

Familia y universidad son instituciones ambas que requieren mucho tiempo y esfuerzo. Para sobrevivir profesionalmente en la Universidad se exige el desarrollo de una carrera; es decir, una secuencia de promociones basadas en méritos, y ello exige un compromiso y una dedicación que entra necesariamente en conflicto con el compromiso y la dedicación que la vida familiar reclama. De acuerdo a las vivencias de las participantes en el estudio, la dificultad más importante reside en la temporalización de los ciclos familiares y profesionales: 'La carrera profesional para las mujeres debe tener un tiempo diferente que para el de los hombres porque el momento más fuerte de producción y de publicación es el momento que tienen los hijos pequeños'. (ent. 011, 128-132).

En el caso de los cargos académicos, esta dificultad se agrava considerablemente. Todas las participantes, sin excepción, han manifestado que tratar de compatibilizar las exigencias de dedicación que se derivan del cargo académico con las demandas familiares les supone un esfuerzo importante. De hecho, el argumento más reiterado por estas mujeres es que, a diferencia de sus compañeros, sólo han podido acceder a estos cargos en un determinado momento de su ciclo vital, una vez las circunstancias familiares les han permitido una dedicación menos exclusiva, especialmente cuando los hijos han alcanzado una determinada edad o bien han abandonado ya el núcleo familiar. Afirmaciones como la de una de las directoras de departamento entrevistadas, que aseguraba que 'lo de directora del departamento no lo podría haber hecho con los niños en casa' (ent. 014, 467-468), o la de una vicerrectora, que aclaraba que 'nunca lo hubiera hecho (aceptar el cargo) antes de que mí hijo tuviera siete años' (ent. 018, 10291030), son compartidas por otras muchas participantes con independencia del cargo o el contexto en que se encuentren. Similarmente, muchas de estas mujeres han señalado la importancia de recibir algún tipo de apoyo o corresponsabilidad por parte de su pareja en la asunción de tareas domésticas y/o educativas.

Muchos de los testimonios aportados por estas mujeres, de hecho, sugieren que las auténticas dificultades a que han debido hacer frente a lo largo de su trayectoria profesional se relacionan con moverse en 'un mundo de hombres' (ent. 013, 817) donde el desarrollo y la valía son enjuiciados de acuerdo con estándares masculinos.

Una de estas asunciones que conforman tácitamente la cultura masculina universitaria es que para las mujeres el desarrollo de la carrera profesional tiene un carácter secundario. Esta asunción, que reiteran con varios matices e intensidades algunas participantes, tiene un elemento común: la convicción por parte de muchos colegas hombres -y de algunas mujeres- de que la necesidad de consolidar una carrera profesional es más fuerte en el hombre que en la mujer.

Una participante recordaba las dificultades que experimentó a lo largo de su carrera mientras contemplaba que sus colegas progresaban a un ritmo más acelerado y se lamentaba: 'Por ejemplo, profesores que me han ayudado mucho a nivel de investigación -de enseñarme a investigar, que son personas de mucha relevancia- en situaciones, por ejemplo, de concursos, que son unos momentos muy duros, me han dicho: Bueno, no te preocupes, ya te Ilegará. Tú ahora dedícate a tus hijos'. (ent. 015, 121-127). 
Otra asunción que regula tácitamente la vida académica consiste en que las mujeres deben demostrar una valía que en los hombres se presupone. Como reflexionaba una de las entrevistadas, 'normalmente el margen de confianza de una mujer es mucho menor: primero se la mide como mujer, y después en función de lo que hace' (ent. 018, 642-644).

En el caso de los cargos académicos, esta asunción se relaciona con la carencia de cualidades para el liderazgo y la gestión universitaria. Una de las vicerrectoras entrevistadas planteaba que esta asunción se rige por una lógica del tipo: 'es vicerrector, luego debe valer, por eso lo han elegido; es vicerrectora, vamos a ver qué hace' (ent. 018, 634-636).

Algunas de las profesoras entrevistadas se han referido también al modo en que operan determinadas redes informales de poder, reforzando la posición de marginalidad de las mujeres y exacerbando su sentimiento de pertenecer a 'otro' colectivo profesional, de orden menor, dentro de la universidad. Dos expresiones comunes en las que se manifiestan estas redes informales es a través de la territorialidad y el acceso a la información.

Las dificultades internas para el desarrollo profesional se refieren, fundamentalmente, a las limitaciones impuestas por los roles de género que las mujeres han interiorizado, pero también se relacionan con la expresión de unos valores distintos y con el deseo de participar profesionalmente en la vida universitaria de forma diferente.

Algunas entrevistadas, por ejemplo, se han mostrando conscientes de los condicionantes sociales que han interiorizado a consecuencia de los roles y los estereotipos asociados al género. Una de las participantes hacía una referencia explícita a estos condicionantes de rol mientras valoraba las circunstancias que han acompañado a su desarrollo profesional: 'Yo no soy de estas personas que dicen que por el hecho de ser mujer me he sentido marginada, pero sí que tengo unos condicionantes de rol que llevo muy adentro que quizás son los que me han hecho tomar esta decisión de no escalar'. (ent. 002, 672-676).

Estos condicionantes se relacionan con una serie de estereotipos que suelen dibujar una imagen socialmente aceptada de la mujer nutrida de características tales como la voluntad de servicio, la orientación hacia conductas cooperativas más que competitivas, y la ausencia de una marcada ambición de poder. Siguiendo un discurso coherente con esta descripción, algunas participantes reflexionaban en las entrevistas sobre el conflicto que se produce entre el espíritu altamente competitivo y la fuerte presión social que ejerce la cultura académica universitaria. Una vicerrectora, por ejemplo, evaluaba de esta manera su opción de no aspirar a la condición de catedrática: 'Yo, por ejemplo, no soy catedrática. Pero no lo soy por una manera vital de ser que probablemente el hecho de ser mujer lo hace más, (...) que es haber sido poco competitiva, (...) y en un determinado momento de mi vida vi que la lucha por los méritos y por publicar en los lugares dónde se debe publicar e ir a según a qué congresos no me interesaba. (...) y es posible que si yo hubiera sido un hombre esto no me lo hubiera permitido'. (ent. 011, 62-70; 74-77).

Estos condicionantes de rol tienen una traducción importante en los cargos de gestión, en la medida en que algunas de las entrevistadas relacionaban la menor presencia 
de las mujeres en lugares de primera responsabilidad con una carencia de ambición de poder, que 'a veces va más en el género masculino' (ent. 002, 178). De acuerdo con esta percepción, pues, las mujeres tienden a mostrarse 'menos ambiciosas a la hora de los cargos grandes, de ser decana de una facultad y rectora' (ent. 013, 644-645). Una de las participantes, que en la actualidad ocupa una responsabilidad académica de primer orden en la universidad donde trabaja, valoraba esta interpretación de la siguiente manera: 'Nosotras las mujeres, en principio, no tenemos una ambición del poder, por llegar. Si me preguntas que quería ser, yo creo que yo quería ser profesora. Punto. Nunca me he planteado en mi vida ser otra cosa que profesora. (...). El que quiere ser se lo trabaja. Las mujeres no lo hacemos. No conozco a ninguna mujer que trabaje por ser decana, y los hombres $\mathrm{si}^{\prime}$. (ent. 004, 222-225; 230-233).

Varias participantes relacionaban estas conductas, además, con la carencia de seguridad y confianza que las mujeres tienen en sí mismas y que también forman parte de los atributos que conforman el estereotipo de género femenino. Y otra remarcaba la importancia de superar estas interiorizaciones de debilidad, desde el convencimiento personal de que 'podemos hacer exactamente lo mismo' (ent. 005, 104).

Pero junto a estas restricciones que imponen los condicionantes de rol de género que las mujeres tienden a interiorizar, su misma condición diferente les permite generar y participar de una serie de valores alternativos a los dominantes. En este sentido, muchas participantes han argumentado que las dificultades al desarrollo profesional y, especialmente, a la asunción de cargos de responsabilidad académica son producto de una auto-restricción que ellas mismas se han impuesto y que las arraiga en la divergencia entre sus propios valores y los de la cultura universitaria dominante.

Buena parte de las entrevistadas coinciden en considerar el sentido de responsabilidad y la voluntad de incidir en la mejora del contexto académico más próximo como las motivaciones que resultan más decisivas para asumir este tipo de funciones. Este argumento, que hemos explorado con más detalle al analizar las circunstancias de acceso a la gestión de las mujeres participantes en este estudio, era considerado por una de las directoras cuando afirmaba: 'quizás la responsabilidad deberá pesar más que el hedonismo, en el buen sentido de la palabra' (ent. 001,786-787).

Finalmente, la disonancia cultural que experimentan algunas participantes se relaciona con su rechazo a los modelos de liderazgo tradicionales, sustentados en un sistema de relaciones jerárquicas que atribuye una serie de privilegios a determinados individuos en virtud de su estatus académico.

Con respecto al objetivo 'Explorar las percepciones de las directivas sobre su comportamiento de liderazgo y los efectos que éste produce', las percepciones que han ofrecido las participantes a este respeto no son unánimes, y pueden representarse en un espectro que oscila entre la negación de las diferencias de género junto con la percepción del estilo de dirección como una cuestión individual en un extremo, y la afirmación de la existencia de una manera distintiva y claramente identificable de ejercer el liderazgo por parte de las mujeres, en el otro.

En todo caso, la mayoría de las participantes han reconocido un discurso popular que atribuye a las mujeres una manera distintiva de ejercer el liderazgo, que 'es más participativo, más hablado' (ent. 008, 427). 
En cambio, las mujeres rara vez se han mostrado taxativas en sus discursos, sugieren más bien que 'la división entre hombres y mujeres no es perfecta' (ent. 008, 517-518), y que el desarrollo de un estilo de liderazgo diferenciado 'es difícil asociarlo con el género en sî́ (ent. 014, 558). De hecho, la mayoría apoya la visión, de manera explícita o implícita, de que aunque hay rasgos diferenciales en los estilos de liderazgo centrado en el poder y orientado a compartir el poder, y entre maneras ejecutivas y consultivas, el género no constituye por si mismo un predictor simple de estos. En otras palabras, su visión es que algunos hombres y mujeres operan en regímenes relativamente democráticos y de toma de decisiones participativa, mientras que otros hombres y mujeres no. El género, en este caso, puede indicar una tendencia, pero en ningún caso una relación de tipo causal.

Respecto al objetivo 'Describir las destrezas y los atributos de liderazgo que las mujeres que lo ejercen consideran más importantes para su trabajo', las mujeres que participan en este estudio señalan rasgos comunes que se caracterizan por representar una diferencia cualitativa respeto a las formas tradicionales de ejercer el liderazgo en la universidad, a saber: una preocupación genuina por atender las necesidades de las personas y desarrollar un clima positivo; un fuerte compromiso con el trabajo en equipo y la participación; y cierta ambivalencia en torno a cuestiones de poder y autoridad. A continuación analizamos estos rasgos tal y como han sido articulados por las participantes en las entrevistas mantenidas.

Una de las directoras, por ejemplo, definía su trabajo como un trabajo 'de puertas adentro', orientado fundamentalmente a cuidar 'de las personas individualmente' (ent. 013, 775-776), hacer 'de relaciones públicas', 'dinamizar las relaciones' y 'limar asperezas' (ent. 013, 351; 382; 386). Otra de ellas describía su estilo de dirección como preocupado por 'hacer que la gente se sienta bien, que cuando han hecho una cosa, se reconozca su esfuerzo' (ent. 014, 552-554). Una tercera resumía su proyecto de dirección como 'un proyecto (..) más de tipo humano' (ent. 015, 836-837). Todavía otra subrayaba la importancia de la empatía en desempeñar un cargo académico: 'Debes ser muy empático en estos cargos. Te debes poner mucho en la piel de los otros' (ent. 012, 265-266).

En relación a este interés por las personas se encuentra también la visión, hazaña explicitada por muchas entrevistadas, de que favorecer un 'buen ambiente para trabajar' (ent. 016, 522) y 'que la gente trabaje más a gusto' (ent. 004, 530) constituyen condiciones necesarias. La preocupación por desarrollar un clima de trabajo positivo aparece unida a la percepción de su estilo de liderazgo orientado a las personas y a las relaciones.

Esta centralidad del componente relacional asociada al liderazgo comporta un cierto elemento de invisibilidad que algunas entrevistadas han relacionado con 'un tipo de comportamiento femenino' (ent. 011, 83). En este sentido, varias de las participantes han señalado que su opción de actuar como 'vaselina social' (ent. 011, 78) comporta un trabajo más de fondo, de sostenimiento de las relaciones, que con frecuencia trae implícita una preferencia por situarse en un segundo plano. Así lo expresaba una de las participantes: '(...) hacíamos un trabajo femenino, (...) de hacer como una red por dentro, de intentar que todo el mundo esté bien, de intentar que haya siempre buen rollo, aunque estés cansada o aunque estés nerviosa' (ent. 012, 194; 199-202).

En segundo término, cuando las participantes reflexionaban sobre las prioridades que guían su estilo de gestión, emergía con fuerza una clara orientación al trabajo en 
equipo y la búsqueda de consenso. El valor del equipo y la colaboración adquiere centralidad en los discursos que las participantes ofrecen sobre sus respectivas experiencias en la gestión universitaria, y se expresa en declaraciones como reconocer que se es 'una persona de equipo' (ent. 015, 1019), creer 'enormemente en el trabajo en equipo' (ent. 002, 518), no hablar 'nunca en primera persona' (ent. 004, 504), o priorizar que 'los equipos funcionen como personas'. (ent. 003, 174-175).

De manera similar, las entrevistadas destacan en varias ocasiones el valor de la transparencia frente a la opacidad que en ocasiones ha caracterizado las dinámicas internas de toma de decisiones en los departamentos u otros contextos universitarios. Algunas participantes han hecho explícita, en este sentido, su preocupación porque durante su gestión 'todo sea muy transparente' (ent. 018, 978). Una de ellas relataba así el cambio que ha querido promover en su departamento en relación con este propósito de transparencia: 'Ahora mis compañeros de departamento saben que realmente lo que explico en la junta, en el consejo, es lo que hay, y que no hay cosas desconocidas. Y se sienten más cómodos, están más tranquilos'. (ent. 015, 1033-1036).

Finalmente, otra temática hace referencia a los sentimientos de ambivalencia que experimentan ante el poder. De hecho, como argumentos en la revisión de la literatura, buena parte de la investigación sobre liderazgo femenino se ha centrado en cuestiones de poder y autoridad, poniendo de manifiesto los sentimientos ambivalentes que las mujeres, que culturalmente han ostentado poco poder, expresan hacia éste. Las entrevistas realizadas contienen indicadores sobre la manera en que estas mujeres tratan de acomodar el poder de una manera efectiva y confortable en el ejercicio del liderazgo. La ambivalencia que han manifestado las participantes al respecto se refleja, fundamentalmente, en la satisfacción que experimentan ante la posibilidad de hacer que las cosas cambien y funcionen mejor, y en el temor que les produce incurrir en un potencial abuso de poder.

El primero de estos aspectos está relacionado con una visión del cargo como 'poder para', esto es, como una capacidad, energía o potencial antes que como fuerza o habilidad por dominar. En este sentido, las mujeres entrevistadas se han referido en varias ocasiones a la autoridad que se deriva del cargo que ocupan en términos de una capacidad que se les otorga para realizar cosas. Cuando este margen real de actuación no existe o es coartado por terceras personas; cuando el ejercicio del cargo se convierte en 'estar allá de figura de paja' (ent. 016, 597) o en 'seguir a pies juntillas todo lo que dijera otra persona' (ent. 009, 764-765), entonces tratan de buscar una redefinición de la situación o, simplemente, abandonan el cargo.

El segundo de los aspectos es 'poder con' en oposición a un 'poder sobre'. La ambivalencia, en este caso, se manifiesta de varias maneras. Por un lado, las entrevistadas tienden a desconfiar y rechazar el poder, especialmente cuando se relaciona con una imposición unilateral. Las definiciones que formulan de sí mismas y del cargo que desarrollan guarda relación con esta negación del poder entendido en términos impositivos. En cargos de mayor responsabilidad, esta consideración se reitera: 'no me gusta decir lo que se debe hacer' (ent. 007, 352-353), declaraba una vicerrectora; 'yo no veo un gran poder y una gran autoridad' (ent. 004, 405-406), confesaba otra participante en un lugar similar.

Por otro lado, frente a una definición del poder en términos impositivos, las participantes han priorizado una comprensión del mismo en términos de capacidad de per- 
suasión. Aunque la búsqueda del consenso, como hemos dicho, constituye un rasgo destacado en la imagen que estas mujeres construyen de sí mismas como gestoras, algunas de ellas han mostrado la ambivalencia que les produce conjugar una actitud de apertura y escucha con la necesidad de reafirmar ciertos planteamientos o apuntar hacia una dirección concreta.

Finalmente, la ambivalencia que experimentan como resultado de su visión de un 'poder con' tiene relación también con la incomodidad que les produce, a varios niveles, convivir con redes informales de poder, o como lo expresaba una de las participantes, con 'una jerarquía académica, que domina sobre la jerarquía de los cargos' (ent. 013, 336-337). Los ejemplos de territorialidad masculina a los que nos referimos al analizar las dificultades internas del desarrollo profesional de la mujer ilustran bien esta idea. En un sentido más amplio, este rechazo se relaciona con su percepción de la universidad como institución con una 'estructura (...) muy jerárquica, donde los catedráticos son señores feudales, y a veces se pasan muchísimo’ (ent.017, 209-210).

\section{Conclusiones}

Constatamos que en la distribución de cargos en las cuatro universidades estudiadas, las mujeres siguen siendo una minoría en los puestos de poder, sobre todo en aquellos de mayor rango. Solamente una de las cuatro universidades está dirigida por una rectora y los vicerrectores continúan estando en manos masculinas en un $80 \%$.

Sobre las dificultades, también Ilamadas en la literatura "barreras", que las profesoras perciben o encuentran en su desarrollo profesional y, en concreto, en el ejercicio del liderazgo, podemos clasificarlas en dos tipos: las dificultades externas a las mujeres y las que son internas. Las primeras harían referencia a lo que está socialmente construido sobre el contenido del rol femenino y las segundas serían derivadas del proceso de socialización de las propias mujeres.

En nuestro estudio las dificultades externas que se han manifestado han sido dos. La primera de ellas es el conflicto de rol en el que entran las mujeres cuando han de elegir entre priorizar su carrera profesional o su vida personal en relación con la capacidad para procrear, ya que ésta requiere un momento madurativo físico y psíquico que suele coincidir en el tiempo con el período que exige una máxima dedicación a la carrera profesional. Tanto los resultados derivados del cuestionario como los derivados de las entrevistas nos muestran que éste motivo sigue siendo el principal limitador para que las mujeres se planteen acceder a cargos de poder en un momento de su trayectoria profesional en que estarían preparadas para ello, como muestran estarlo sus colegas masculinos.

La segunda dificultad es la cultura organizativa predominante en la organización, basada principalmente en valores tradicionalmente asociados a lo masculino. Las profesoras participantes en nuestra investigación afirman no sentirse a gusto con los valores y las estrategias de acción existentes en los órganos de gobierno de sus universidades, por el hecho de sentirlos ajenos a ellas. De hecho, como hemos visto, las cifras constatan que no ha habido muchas mujeres que hayan participado en su formación. Así, muchas de las profesoras no se sienten suficientemente identificadas con la cultura de la institución para desear estar al frente de sus órganos de gestión. El rechazo a los modelos de 
liderazgo predominantes tradicionalmente considerados masculinos, las desmotiva para postularse como candidatas al ejercicio de cargos.

La principal dificultad que podemos considerar interna consiste en la falta de interés por el poder que manifiestan las profesoras entrevistadas. También coinciden todas las participantes en afirmar que ocupar un lugar de poder sirve para "poder hacer algo", "poder cambiar algo". Así, nos parece que debería matizarse la creencia, muy extendida por cierto, de que las mujeres no están interesadas en ejercer poder. De nuestro estudio se desprende que lo que desprecian es el "poder sobre alguien" pero, en cambio, manifiestan apreciar el "poder para algo" y el "poder con alguien".

Tabla 1. Ejercicio de cargos académicos según cargo, universidad y sexo

\begin{tabular}{|c|c|c|c|c|c|c|c|c|}
\hline \multirow{2}{*}{$\begin{array}{l}\text { Universidad } \\
\text { Cargos }\end{array}$} & \multicolumn{2}{|c|}{ U-1 } & \multicolumn{2}{|c|}{ U-2 } & \multicolumn{2}{|c|}{ U-3 } & \multicolumn{2}{|l|}{$U-4$} \\
\hline & mujeres & hombres & mujeres & hombres & mujeres & hombres & mujeres & hombres \\
\hline Rector/a & 0 & 1 & 0 & 1 & 0 & 1 & 1 & 0 \\
\hline$\%$ & 0 & 100 & 0 & 100 & 0 & 100 & 100 & 0 \\
\hline Vicerector/a & 6 & 4 & 2 & 7 & 2 & 8 & 1 & 2 \\
\hline$\%$ & 60 & 40 & 22.22 & 77.78 & 20 & 80 & 25 & 75 \\
\hline Decano/Decana & 3 & 8 & 5 & 13 & 1 & 8 & 0 & 8 \\
\hline$\%$ & 27.27 & 72.73 & 27.77 & 72.73 & 11.11 & 88.89 & 0 & 100 \\
\hline Director/a & 1 & 2 & 2 & 0 & 1 & 3 & 37 & \\
\hline $\begin{array}{l}\text { Escuela } \\
\%\end{array}$ & 33.33 & 66.67 & 100 & 0 & 25 & 75 & 30 & 70 \\
\hline Director/a & 12 & 42 & 26 & 77 & 1 & $8(\sin c$ & latos) & \\
\hline Departamento & & & & & & & & \\
\hline$\%$ & 22.22 & 77.78 & 25.24 & 74.76 & 11.11 & 88.89 & & \\
\hline
\end{tabular}

\section{Referencias bibliográficas}

Acker, J. (2000). "Jerarquías, trabajos y cuerpos: una teoría sobre las organizaciones dotadas de género. En: M. Navarro y C. R. Stimpson (comp..): Cambios sociales, económicos y culturales. (111-139). México, FCE.

Alimo-Metcalfe, B. (1995). "An investigation of female and male constructs of leadership and empowerment", Women in Management Review, 10(2): 3-8.

Alvesson, M. \& Billing, Y.D. (1992). "Gender and organization: Towards a differentiated understanding", Organization Studies, 13/12: 73-102.

Alvesson, M. \& Billing, Y.D. (1997). Understanding gender and organizations. Londres, Sage.

Appelbaum, S.H., Audet, L. \& Miller, J.C. (2003). "Gender and leadership? Leadership and gender? A journey through the landscape of theories", Leadership \& Organization Development Journal, 24(1): 43-51. 
MARINA TOMÀS FOLCH, MARIA DEL MAR DURAN BELLONCH, CRISTINA GUILLAMÓN RAMOS, JOSÉ MANUEL LAVIÉ MARTÍNEZ

Billing, Y.D. y Alvesson, M. (2000). "Questioning the notion of feminine leadership: A critical perspective on the gender labeling of leadership", Gender, Work and Organization, 7(3): 144-157.

Blackmore, J. (1996). "Breaking the silence: Feminist contributions to educational administration and policy". En: K. LEITHWOOD, J. CHAPMAN, D. CORSON, P. HALLINGER \& A. HART (eds.) International handbook of educational leadership and administration (pp. 997-1042). Dordrecht, Kluwer Academic Publishers.

Blackmore, J. (1999) Troubling women: Feminism, leadership and educational change. Buckingham, Open University Press.

Burke, S. \& Collins, K.M. (2001). "Gender differences in leadership styles and management skills", Women in Management Review, 16(5): 244-256.

Calás, M.B. y Smircich, L. (2006). "From the 'women's point of view' ten years later: Towards a feminist organization studies". In: S.R. CLEGG, C. HARDY, T.B. LAWRENCE \& W.R. NORD (eds.) The Sage Handbook of Organization Studies (pp. 284-346, 2a edición). Londres, Sage.

Carreño, A., Fabra, M.L., Lloret, C. et al. (1998). La carrera de les profesores universitàries. Barcelona, ICE de la UAB.

Davies, C. \& Holloway, P. (1995). "Troubling transformations. Gender regimes and organizational cultures in the Academy". In: L. MORLEY Y V. WALSH (eds) Feminist academics: Creative agents for change. Londres, Taylor \& Francis.

Díez Guitiérrez, J. E.; Terrón, E.; Anguita, R. (2005). La cultura de género en las organizaciones escolares. Motivaciones y obstáculos para el acceso de las mujeres a los puestos de dirección. Barcelona, Octaedro.

Eagly, A.H. \& Johannesen-Schmidt, M.C. (2001). "The leadership styles of women and men", Journal of Social Issues, 57(4): 781-797.

Eagly, A.H. \& Johnson, B. (1990). "Gender and leadership style: A meta-analysis", Psychological Bulletin, 108(2): 233-256.

Ely, R.J. \& Meyerson, D.E. (2000). "Theories of gender in organizations: A new approach to organizational analysis and change", Research in Organizational Behaviour, 22: 103-151.

Fischer, H. (2000). El primer sexo. Las capacidades innatas de la mujer y cómo están cambiando el mundo. Torrelaguna, Santillana.

Gherardi, S. (1995). Gender, Symbolism and Organizational Cultures. London Sage

Guillamón, C. (2006). El desenvolupament professional, I'assumpció de càrrecs i l'estil de lideratge de les directores de departament a la universitat catalana. Trabajo de investigación inédito, Universitat Autónoma de Barcelona.

Halford, S. \& Leonard, P. (2001). Gender, power and organization. New York, Palgrave.

Hawley, E., Torres, D. \& Rasheed, D. (1998). "Assessing barriers to women's career adjustment", Journal of Career Adjustment, 6(4): 449-479. 
Hudson, M. \& Williamson, R. (2002). "Women transitioning into leadership: Gender and both help and hindrance", Advancing Women in Leadership Journal (http://www.advancingwomen.com)

Hunt, J.G. (1999). "Transformational / charismatic leadership's transformation of the field: An historical essay", Leadership Quarterly, 10: 129-144.

Johnsrud, L.K. (1994). "Enabling the success of junior faculty women through mentoring", New Directions for Teaching and Learning, 57: 53-63.

Kettle, J. (1996). "Good practices, bad attitudes: An examination of the factors influencing women's academic careers". In: L. MORLEY Y V. WALSH (eds.) Breaking boundaries: Women in Higher education (pp. 52-66). Bristol, Taylor \& Francis.

Maddock, S. (1999). Challenging women: gender, culture and organization. Londres, Sage.

Maier, M. (1999). "On the gendered substructure of organization: Dimensions and dilemmas of corporate masculinity", In: G.N. POWELL (ed.) Handbook of gender and work (pp. 69-93). Londres, Sage.

Miles, M. \& Huberman, M. A. (1994). Qualitative data analysis (second edition). Londres, Sage.

Morley, L. (2004). "Women's careers in Higher education: Theorising gender inequalities". Women in European Universities (http://www.women-eu.de/form.htm).

Nicolson, P. (1997). Poder, género y organizaciones. ¿Se valora a la mujer en la empresa? Madrid, Narcea.

Powell, G.N. (1999). "Reflections on the glass ceiling. Recent trends and future prospects". In: G.N. POWELL (ed.) Handbook of gender and work (pp. 325-345). Londres, Sage.

Riehl, C. y Lee, V.E. (1996). "Gender, organizations and leadership". In: K. LEITHWOOD, J. CHAPMAN, D. CORSON, P. HALLINGER \& A. HART (eds.) International handbook of educational leadership and administration (pp. 873-919). Dordrecht, Kluwer Academic Publishers.

Rutherford, S. (2001). "Organizational cultures, women managers and exclusion", Women in Management Review, 16(8): 371-382.

Schein, V.E. (2001). "A global look at psychological barriers to women's progress in management", Journal of Social Issues, 57(4): 675-688.

Van Vianen, A. \& Fischer, A. (2002). "Illuminating the glass ceiling: The role of organizational culture preferences", Journal of Occupational and Organizational Psychology, 75: 315-337. 